\title{
Use of low field NMR for the characterization of gels and biological tissues
}

\author{
Michela Abrami ${ }^{1}$, Gianluca Chiarappa ${ }^{1}$, Rossella Farra ${ }^{1}$, Gabriele Grassi ${ }^{2}$, Paolo \\ Marizza $^{3}$, Mario Grassi ${ }^{*}$ \\ ${ }^{1}$ Department of Engineering and Architecture, Trieste University, Via Valerio 6, Trieste, I-34127, Italy \\ ${ }^{2}$ Department of Life Sciences, Trieste University, Strada di Fiume 447, Trieste, I-34149, Italy \\ ${ }^{3}$ Department of Micro- and Nanotechnology, Technical University of Denmark (DTU), Ørsteds Plads Bygning 345 $\emptyset$, \\ Kongens Lyngby 2800, Denmark
}

*Corresponding Author: E-mail: mario.grassi@dia.units.it; Tel.: +39-040-558-3435; Fax: +39-040-569823

Received: August 30, 2017; Revised: March 16, 2018; Published: March 25, 2018

\begin{abstract}
The focus of this paper is on the theoretical interpretation of Low Field Nuclear Magnetic Resonance (LFNMR) data regarding hydrogels architecture and on the most interesting applications of LF-NMR presented by this research group at the $6^{\text {th }}$ IAPC Symposium held in Zagreb (HR) on September 2017. Particular attention is devoted to the determination of the mesh size distribution of gels polymeric network and the determination of the pore size distribution of microporous systems such as scaffolds, bones, and porous gels. In addition, we report on a very recent application of LF-NMR for monitoring lung functioning in patients suffering from chronic pulmonary diseases like cystic fibrosis. The main findings of this work consist in providing a very simple and accurate approximation of a general theory devoted to evaluating the relation existing among four fundamental polymeric network parameters, i.e. the polymer volume fraction inside the hydrogel, mesh size, hydraulic radius, and the radius of the cylinder ideally embedding each polymeric network chain. Furthermore, we demonstrated the potentiality of LF-NMR in the characterization of different polymeric systems among which the sputum of patients suffering from chronic pulmonary diseases appears the most innovative application for its simplicity, rapidity, effectiveness, and potential impact in the everyday clinic.
\end{abstract}

\section{Keywords}

Low Field NMR; mesh size distribution; pores size distribution; biomedical applications

\section{Introduction}

Despite the widespread use of high-field nuclear magnetic resonance $(7-37 \mathrm{~T})$ for the study of chemical structures, low-field nuclear magnetic resonance (LF-NMR; $0.37-2.4 \mathrm{~T}$ ) is quite uncommon. Nevertheless, the fundamental works of Brownstein and Tarr [1,2], Mitra et al. [3], Torrey et al. [4], and Chui et al. [5] clearly demonstrated the potentiality of LF-NMR for the study of different materials. Accordingly, LF-NMR finds interesting applications in many fields like food chemistry. For instance, Hills et al. [6] explored water distribution in skimmed milk and apple, Aroulmoji et al. [7] investigated the hydration properties of aqueous sugar solutions, and De'Nobili et al. [8] delved pectin/alginates films for the release of ascorbic acid in agar hydrogels mimicking food materials. Moreover, LF-NMR proved to be very useful in 
determining the size distribution of oil droplets in oil-water emulsions $[9,10]$, an important aspect for different kinds of edible creams like mayonnaise. At the same time, LF-NMR resulted to be an extremely important characterization tool in the petroleum industry as it may be applied not only to predicting the viscosity of crude oils and crude oil emulsions [11] but also to profitably evaluating the pores size distribution of the rocks containing petroleum [12-14]. The capability of estimating the pore size distribution rendered LF-NMR a valuable approach also in the biomedical field. Indeed, many authors employed LF-NMR to characterize bones [15-18], a very particular porous material, while Grassi et al. [19] and Fiorentino et al. [20] focussed on the characterization of polymeric scaffolds for tissue engineering. Finally, Abrami et al. [21] dealt with a very new application of LF-NMR concerning the characterization of expectorate of patients affected by chronic pulmonary diseases like cystic fibrosis (CF).

Undoubtedly, however, one of the most common LF-NMR applications regards the characterization of hydrogels whose three-dimensional architecture is able to affect water hydrogens magnetic relaxation. For example, Ghi et al. [22] studied the architecture of PHEMA hydrogels, Barbucci et al. [23] considered hyaluronan-based hydrogels, Hietalahti et al. [24] investigated the crosslinking in unsaturated polyester solutions, Brand et al. [25] focussed on the gel point determination of gelatin, Dobies et al. [26] explored the gelation process of aqueous low methoxyl pectin solutions, while Abrami et al. [27] delved the properties of an interpenetrated network made of alginate and Pluronic F127.

Despite the great variety of LF-NMR applications, it is important to remember that, whatever the system considered is, the guiding principle allowing LF-NMR characterization relies on the effect of solid surfaces (polymeric chains, bones, rocks, and others) on the relaxation process of water hydrogens subjected to a sudden variation of an external magnetic field. The higher the ratio between system solid surface and system volume is, the faster is the hydrogens relaxation process. Based on this physical evidence, it is possible to obtain interesting information concerning the three-dimensional architecture of gels network (mesh size distribution) and the pores size distribution of porous materials. The focus of this paper is on 1) some considerations on the theoretical interpretation of LF-NMR data regarding hydrogels architecture (see Theoretical Background section) and 2) the most interesting LF-NMR applications presented by this research group at the $6^{\text {th }}$ IAPC Symposium held in Zagreb (HR) on September 2017 (see Results and Discussion section). In particular, applications concern the characterization of polymeric gels, scaffolds, and biological tissues such as bones and sputum of CF patients.

\section{Theoretical background}

The principle of LF-NMR relies on the effect of a static magnetic field $B_{0}$ on the dipole moments of some atoms like hydrogens (protons). Indeed, hydrogens dipole moments do not align themselves with the magnetic field direction, but their axes start a precession movement around $B_{0}$ direction. This precession is characterized by the resonance frequency $v_{0}$ and the angle $(\theta)$ formed between dipole moments and $B_{0}$ direction. As all dipole moments react in the same manner to the presence of $B_{0}$, they generate the global magnetization vector $M$, whose components are $M_{z}$, conventionally aligned with $B_{0}, M_{x}$, and $M_{y}$ lying in the $x y$ plane perpendicular to $B_{0}$. Since dipole moments are randomly oriented on $\mathrm{x}$ and $\mathrm{y}$ axes, they cancel themselves and the overall $M_{x y}=0$ (no phase coherence). In addition, as some dipole moments are able to start a precession movement around the direction opposite that of $B_{0}$ (but they are the minority for energetic reasons), $M_{z}$ module depends on the difference between the parallel and antiparallel oriented dipole moments. The application of a radiofrequency field $B_{1}$, rotating at the resonance frequency and normal to $B_{0}$, allows the rotation of $M$ around $B_{1}$ of the angle $\Delta \theta=\gamma^{*} B_{1}{ }^{*} t_{p}$, where $\gamma$ is the gyromagnetic ratio (whose value depends on the particular atom) and $t_{\mathrm{p}}$ is the pulsation time of $B_{1}$. When $\Delta \theta=90^{\circ}, M_{\mathrm{xy}}=$ 
$M_{0}$ and $M_{z}=0$. Thus, upon $B_{1}$ removal just after $t_{p}$, all dipole moments will return to their original orientation ( $M$ parallel to $B_{0}$ ) by rotating around $B_{0}$ with a decreasing angle up to the attainment of $\theta$. This re-orientation implies $M_{\mathrm{xy}}$ reduction and $M_{z}$ increase up to $M_{\mathrm{xy}}=0$ and $M_{z}=M$. The parameter used to evaluate the speed of $M_{x y}$ disappearance is a constant called average relaxation time $T_{2 m}$ (spin-spin relaxation time). At the same time, the increase of the $M_{z}$ component is characterized by another average relaxation time $\left(T_{1 \mathrm{~m}}\right)$ called spin-lattice relaxation time. The ratio $M_{\mathrm{xy}} / M$ is called FID (free induction decay). FID $(=I(t))$ temporal evolution may be described according to the following sum of exponential functions [5]:

$$
I(t)=\sum_{i=1}^{m} A_{i} \exp \left(-t / T_{2 i}\right) \quad T_{2 \mathrm{~m}}=\sum_{\mathrm{i}=1}^{\mathrm{m}} A_{\mathrm{i}} T_{2 \mathrm{i}} / \sum_{\mathrm{i}=1}^{\mathrm{m}} A_{\mathrm{i}} \quad\left(\frac{1}{T_{2}}\right)_{m}=\sum_{i=1}^{n} \frac{A_{i}}{T_{2 i}} / \sum_{i=1}^{n} A_{i}
$$

where $t$ is time, $A_{\mathrm{i}}$ are pre-exponential factors (dimensionless), proportional to the number of protons relaxing with the relaxation times $T_{2 i}$ and $T_{2 m}$, is the average relaxation time of protons.

\section{CPMG sequence}

Unfortunately, due to the unavoidable presence of magnetic field inhomogeneities, after the application of the $B_{1}$ pulse, magnetization $M$ undergoes the fanning-out phenomenon consisting in the clockwise and anticlockwise rotation of the faster and the slower $M$ components. Indeed, $M$ is owed to dipole moments rotating with different frequencies due to the above-mentioned field inhomogeneities. Ultimately, fanningout makes virtually impossible FID determination. In order to properly overcome the fanning-out effect, the first $B_{1}$ pulse (provoking a $\pi / 2$ rotation of $M$ ) is followed, after a time $\tau$, by a second $B_{1}$ pulse causing a further $M$ rotation of $\pi$. By doing so, the faster and the slower $M$ components invert their rotation direction thus meeting after a time of $2 \tau$ from the first $B_{1}$ pulse, when it is possible to measure FID. The sequence $90^{\circ}-\tau-180^{\circ}-\tau$ (echo) is called Hahn spin-echo sequence. In order to follow the entire relaxation process, it is necessary to apply $n$ times the $B_{1}$ pulse (each one separated by a time interval $\tau$ ) thus provoking a $\pi$ rotation of $M: 90^{\circ}\left[-\tau-180^{\circ}-\tau \text { (echo) }\right]_{n}$. This is the so-called CPMG (Carr-Purcell-Meiboom-Gill) pulse sequence [28].

\section{Surface effect on $T_{2 m}$}

$T_{2 \mathrm{~m}}$ depends on many factors such as temperature, magnetic field intensity, and the interaction among hydrogens and other possible substances present in the environment [29]. In particular, hydrogens interactions with solid surfaces (for instance dispersed/solubilized polymeric chains and system boundaries) are one of the most important causes of $T_{2 \mathrm{~m}}$ variation. Indeed, the relaxation time of water protons near the solid surface (bound water protons) is lower (fast relaxation) than that (slow relaxation) of bulk water protons (free water protons) that are unaffected by solid surface [5]. Accordingly, the average relaxation time $\left(T_{2 \mathrm{~m}}\right)$ of protons will depend on the ratio between the solid surface area $(S)$, proportional to the number of protons close to $S$, and system volume $(V)$, proportional to the total number of protons belonging to the system, as demonstrated by Brownstein and Tarr [1] in the case of solid porous systems. In particular, the relation between $S, V$, and the average relaxation time may be represented by [29]:

$$
\left(\frac{1}{T_{2}}\right)_{m}=\frac{1}{T_{2 \mathrm{H} 2 \mathrm{O}}}+\frac{S}{V} \frac{\lambda}{T_{2 \mathrm{~S}}}
$$

where $T_{2 \mathrm{H} 2 \mathrm{O}}$ is the bulk protons relaxation time (i.e. the free water protons relaxation time $T_{2 \mathrm{H} 2 \mathrm{O}} \approx 3700 \mathrm{~ms}$ at $37{ }^{\circ} \mathrm{C}, 20 \mathrm{MHz}$ [30]), $\lambda$ is the thickness of the water layer close to the solid surface (bound water), while $T_{2 s}$ is the relaxation time of bound water protons. Equation (2) clarifies that, when the ratio $S / V$ rises, 
$\left(1 / T_{2}\right)_{m}$ increases and $T_{2 m}$ decreases. Indeed, relying on the Equation (1), it follows that $\left(1 / T_{2}\right)_{m} \neq 1 / T_{2 m}$, but it is easy to mathematically verify that, when $\left(1 / T_{2}\right)_{\mathrm{m}}$ increases, $T_{2 \mathrm{~m}}$ decreases. As the evaluation of $T_{2 \mathrm{~s}}$ is very complex, by starting from Equation (2), Chui and co-workers [5] derived the following relation:

$$
\left(\frac{1}{T_{2}}\right)_{m}=\frac{1}{T_{2 \mathrm{H} 2 \mathrm{O}}}+2 \frac{\mathcal{M}}{R_{h}}, \quad R_{\mathrm{h}}=2 \frac{V}{S},
$$

where $R_{\mathrm{h}}$ is the system hydraulic radius, while $\mathcal{M}$ is a physical parameter (relaxivity, length/time) considering the surface effect on proton relaxations. Indeed, $\mathcal{M}$ represents the ratio between the thickness and the relaxation time of the bound water layer adhering to the solid surface (thus $\mathcal{M}=\lambda / T_{2 s}$ ). $\mathcal{M}$ depends not only on the solid surface chemistry but also on temperature, hydrogenated fluid type, and magnetic field strength [29]. Typically, at $20 \mathrm{MHz}$, in the temperature range $25-37{ }^{\circ} \mathrm{C}$, polymeric materials are characterized by $\mathcal{M}$ values ranging between $10^{-7}-10^{-5} \mathrm{~m} / \mathrm{s}$ [31]. In the case of nano-structured systems like gels, Chui [5] expressed the hydraulic radius as a function of three very important network parameters, i.e. the average mesh size $\xi_{\mathrm{a}}$, the polymer volume fraction $(\phi)$, and the radius $\left(R_{\mathrm{f}}\right)$ of the cylinder ideally containing the single polymeric chain of the gel network: $R_{\mathrm{h}}=f\left(\phi, \xi_{\mathrm{a}}, R_{\mathrm{f}}\right)$. Accordingly, Equation (3) becomes:

$$
\left(\frac{1}{T_{2}}\right)_{m}=\frac{1}{T_{2 \mathrm{H} 2 \mathrm{O}}}+2 \frac{\mathcal{M}}{f\left(\xi_{a}, \phi, R_{f}\right)} .
$$

While Chui's expression [5] for $f\left(\phi, \xi_{\mathrm{a}}, R_{\mathrm{f}}\right)$ relies on the assumption that the polymeric network is nothing but a very long fiber, Scherer [32], by providing a more sophisticated and physically sound schematization of the polymeric network (cubic, octahedral or tetrahedral spatial arrangement), obtained a different expression for $f\left(\phi, \xi_{a}, R_{f}\right)$. Nevertheless, also in the simplest case of a cubic arrangement, $f\left(\phi, \xi_{a}, R_{f}\right)$ is, mathematically speaking, not particularly user-friendly. As a result, Scherer [32] proposed the following empirical approximation holding whatever the network arrangement considered is (cubic, octahedral or tetrahedral):

$$
R_{h}=R_{f} \frac{1-0.58 \varphi}{\varphi}
$$

This expression ensures a very good approximation of the original Scherer model (relative error $<10 \%$ whatever the network spatial arrangement is) for $\phi \leq 0.56$, a very high value that is never encountered in real gels. Equation (5) represents an optimal starting point to deduce a useful approximate relation among $\xi_{\mathrm{a}}, \phi$, and $R_{\mathrm{f}}$. Indeed, the original Scherer equation [32] yields the following cubic equation in $\xi_{\mathrm{a}}$ :

$$
\xi_{a}^{3}\left(\frac{2 C_{0}}{R_{h} R_{f}}\right)-2 C_{1} \xi_{a}\left(1+\frac{R_{f}}{R_{h}}\right)+C_{2} R_{f}\left(2 \frac{R_{f}}{R_{h}}+3\right)=0,
$$

where $C_{0}, C_{1}$, and $C_{2}$ are constants depending on the specific spatial arrangement of the network (see Table 1).

As, in the light of Equation (5), we may assume that $R_{\mathrm{f}} / R_{\mathrm{h}} \approx 0$ (at least for $\phi \leq 0.1$ ), Equation (6) may be re-written as:

$$
\xi_{a}^{3}\left(\frac{2 C_{0}}{R_{h} R_{f}}\right)-2 C_{1} \xi_{a}+3 C_{2} R_{f}=0,
$$

or:

$$
R_{h}\left(\xi_{a}^{3}\left(\frac{2 C_{0}}{R_{h}^{2} R_{f}}\right)-\frac{2 C_{1} \xi_{a}}{R_{h}}+3 C_{2} \frac{R_{f}}{R_{h}}\right)=0
$$


Table 1. Constants of the Scherer's model [32]

\begin{tabular}{|l|c|c|c|}
\hline Network structure & $\mathbf{C}_{\mathbf{0}}$ & $\mathbf{C}_{\mathbf{1}}$ & $\mathbf{C}_{\mathbf{2}}$ \\
\hline Cube & 1 & $3 \pi$ & $8 \sqrt{2}$ \\
\hline Octahedron & $\frac{\sqrt{2}}{3}$ & $12 \arcsin \left(\frac{\sqrt{6}}{3}\right)$ & $16 \sqrt{2}$ \\
\hline Tetrahedron & $\frac{\sqrt{2}}{12}$ & $6 \arcsin \left(\frac{\sqrt{3}}{3}\right)$ & 8 \\
\hline
\end{tabular}

By assuming, again, that $R_{\mathrm{f}} / R_{\mathrm{h}} \approx 0$, Equation (8) becomes:

$R_{h}\left(\xi_{a}^{3}\left(\frac{2 C_{0}}{R_{h}^{2} R_{f}}\right)-\frac{2 C_{1} \xi_{a}}{R_{h}}\right)=R_{h} \xi_{a}\left(\xi_{a}^{2}\left(\frac{2 C_{0}}{R_{h}^{2} R_{f}}\right)-\frac{2 C_{1}}{R_{h}}\right)=0$.

It is easy to observe that, apart from the trivial solution $\left(\xi_{\mathrm{a}}=0\right)$, the only physically sound solution of Equation (9) is:

$$
\xi_{a}=\sqrt{\frac{C_{1}}{C_{0}} R_{h} R_{f}}
$$

By substituting $R_{\mathrm{h}}$ for its approximation (eq.(5)), the final approximate expression for $\xi_{\mathrm{a}}$ is obtained:

$$
\xi_{a}=R_{f} \sqrt{\frac{C_{1}}{C_{0}} \frac{1-0.58 \varphi}{\varphi}} .
$$

On the basis of Equation (11) and Equation (5), we are also able to derive the direct relation between $R_{\mathrm{h}}$ and $\xi_{\mathrm{a}}$ :

$$
R_{h}=\xi_{a} \sqrt{\frac{C_{0}}{C_{1}} \frac{1-0.58 \varphi}{\varphi}}
$$

Thus, Equation (4) may be satisfactorily approximated by:

$$
\left(\frac{1}{T_{2}}\right)_{m}=\frac{1}{T_{2 \mathrm{H} 2 \mathrm{O}}}+2 \frac{\mathcal{M}}{R_{f} \frac{1-0.58 \varphi}{\varphi}} \quad \text { or } \quad\left(\frac{1}{T_{2}}\right)_{m}=\frac{1}{T_{2 \mathrm{H} 2 \mathrm{O}}}+2 \frac{\mathcal{M}}{\xi_{a} \sqrt{\frac{C_{0} 1-0.58 \varphi}{C_{1} \varphi}}} .
$$

In the case of a cubic arrangement of the network (similar considerations also apply in the case of octahedron and tetrahedron), Figure 1 shows the very good agreement between Equation (11) (solid line) and Scherer's original expression (Equation (6), open circles). It is possible to observe (dotted line; right vertical axis) that whatever $\phi$ is, the relative error is always $<5 \%$. Figure 2 demonstrates that a good agreement between Scherer's model (Equation (6), open circles) and our approximation (Equation (12)) occurs (relative \% error $<10 \%$ ) up to $\phi<0.61$.

In the case of porous systems (such as zeolites, porous rocks, and microporous gels), the evaluation of the $S / V$ ratio is considerably simpler if pores are assumed spherical. Indeed, with this hypothesis, $S / V=\xi_{\mathrm{a}} / 3$, so that Equation (3) becomes:

$$
\left(\frac{1}{T_{2}}\right)_{m}=\frac{1}{T_{2 \mathrm{H} 2 \mathrm{O}}}+6 \frac{\mathcal{M}}{\xi_{a}} .
$$

Since $T_{2 \mathrm{H} 2 \mathrm{O}}$ is known and $\left(1 / T_{2}\right)_{\mathrm{m}}$ may be experimentally determined by fitting Equation (1) to experimental relaxation data, Equation (14) may be employed to evaluate $\mathcal{M}$ once $\xi_{\mathrm{a}}$ has been evaluated by an independent approach. Interestingly, $\xi_{\mathrm{a}}$ may be determined resorting to the PGSE sequence (pulsed gradient spin echo) [33] which is the CPMG sequence where two gradient fields are applied after the two pulses $B_{1}(\pi / 2)$ and $B_{1}(\pi)$. The presence of the two gradients of intensity $G$ allows determining the water self-diffusion coefficient $D_{\mathrm{w}}$ dependence on the diffusion time [34]: 


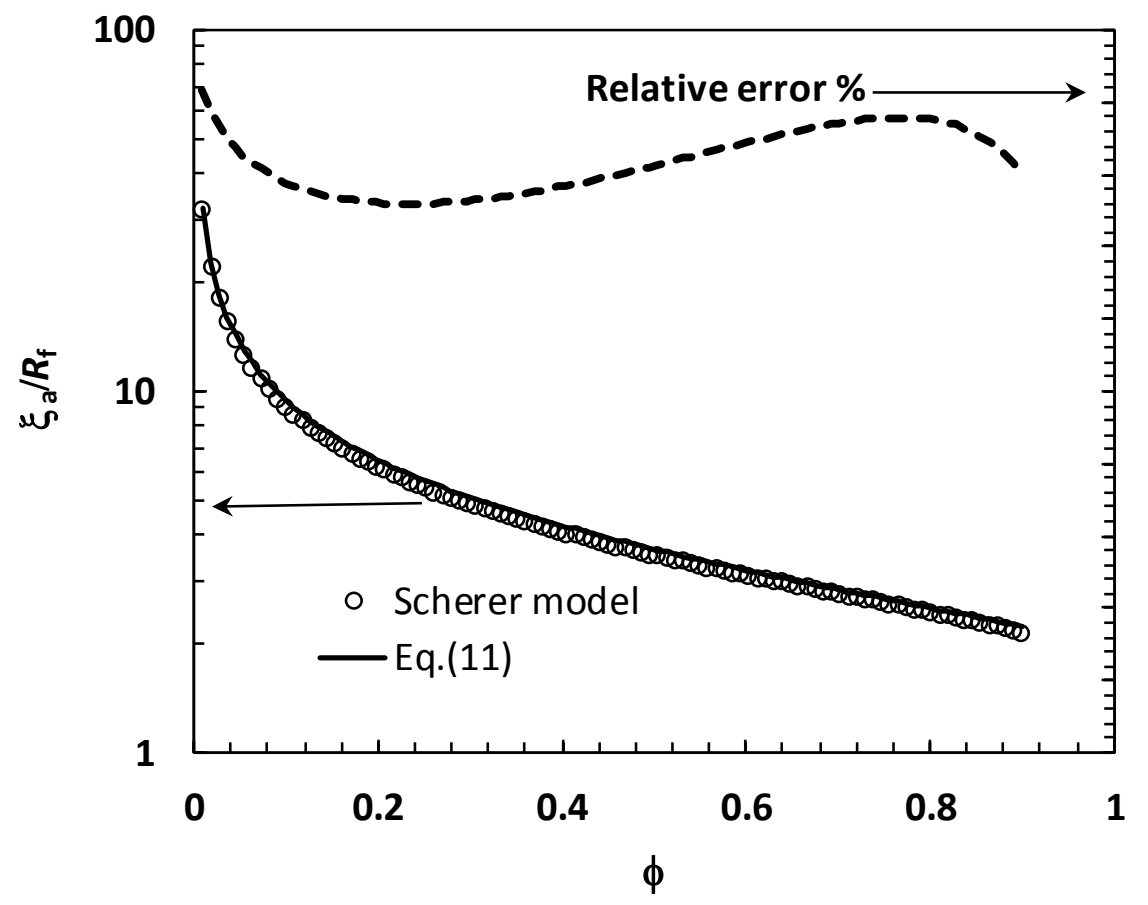

0

$-2$

$-4$

$-6$

$-8$

$-10$

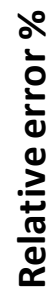

$-16$

$-18$

$-20$

Figure 1. Comparison between Scherer's model (Equation (6), open circles) and our approximation (Equation (11), solid line, cubic arrangement). The dotted line indicates the relative $\%$ error (right vertical axis), $\phi$ is the polymer volume fraction characterizing the network, $R_{\mathrm{f}}$ is the radius of the cylinder able to contain each polymeric chain in its linear configuration, while $\xi_{\mathrm{a}}$ is the average mesh size.

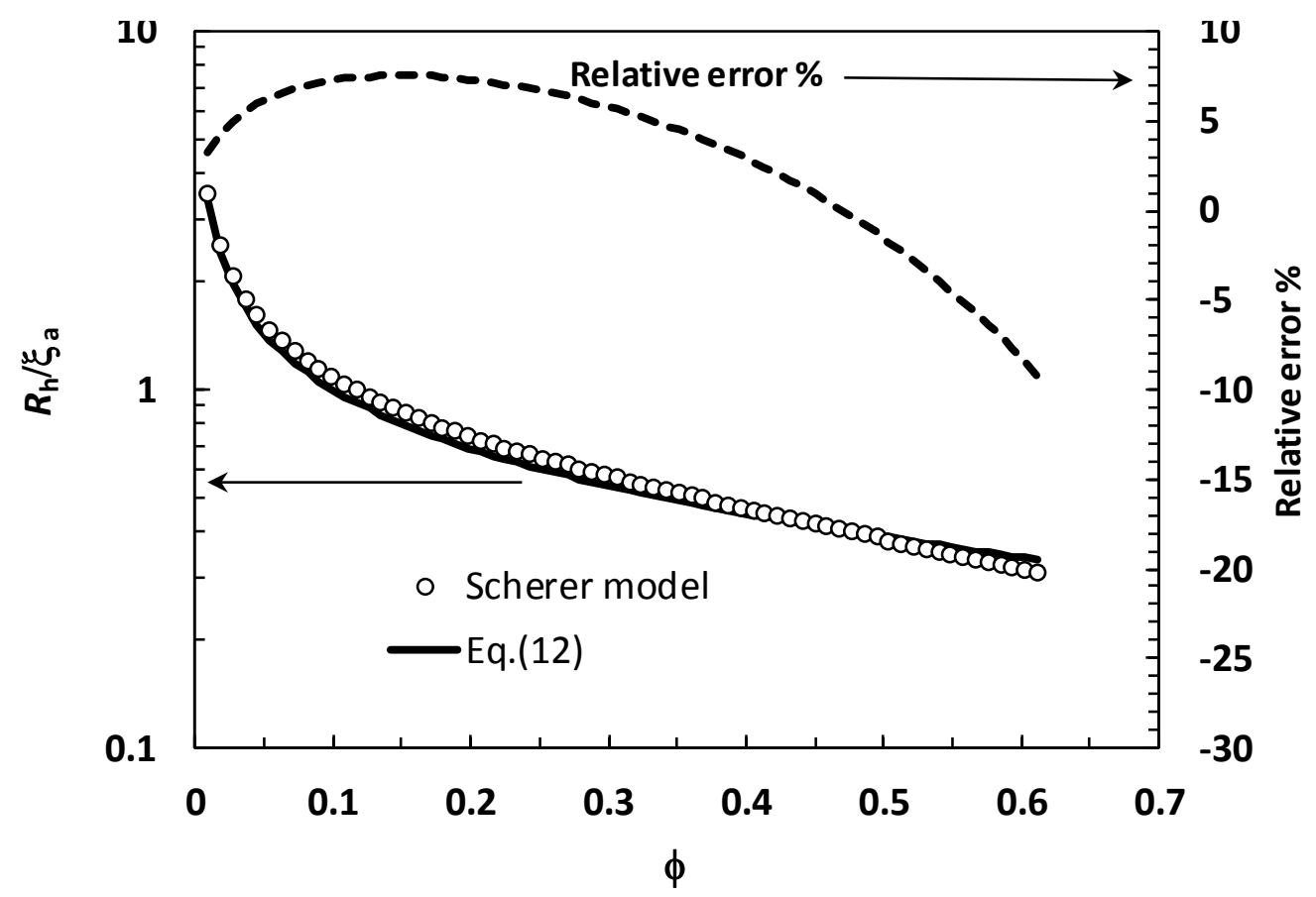

Figure 2. Comparison between Scherer's model (Equation (6), open circles) and our approximation (Equation (12), solid line, cubic arrangement). The dotted line indicates the relative \% error (right vertical axis), $\phi$ is the polymer volume fraction characterizing the network, $R_{\mathrm{h}}$ is the hydraulic radius, and $\xi_{\mathrm{a}}$ is the average mesh size.

$$
\left.\ln \left(\frac{I}{I_{0}}\right)=-\gamma^{2} D_{w} \delta^{2}(\Delta-\delta / 3)\right) G^{2}
$$

where $\gamma$ is the hydrogen gyromagnetic ratio, $\delta$ is the gradient length, $\Delta$ is the time elapsed from the beginning of the first gradient and the one of the second gradient, while $t_{\mathrm{d}}=\Delta-\delta / 3$ is the diffusion time 
(time allowed for hydrogens diffusion). For small $t_{\mathrm{d}}$ values, $D_{\mathrm{w}}$ is connected to $\xi_{\mathrm{a}}$ according to the following equation [35]:

$$
\frac{D_{w}\left(t_{d}\right)}{D_{w f}} \approx 1-\frac{4}{9 \sqrt{\pi}} \sqrt{D_{w f} t_{d}} \frac{6}{\xi_{a}},
$$

where $D_{\mathrm{wf}}$ is the free water self-diffusion coefficient. Fitting Equation (16) to experimental $D_{w}$ vs $t_{\mathrm{d}}$ data allows the determination of $\xi_{\mathrm{a}}$.

\section{Mesh/pore size distribution}

Obviously, a real polymeric network/porous system is composed of meshes/pores of different sizes that may be grouped into different main classes, each one characterized by its proper dimension $\xi_{\text {i. }}$ Interestingly, different $\xi_{\mathrm{i}}$ correspond to different $S / V$ values, i.e. different relaxation times $T_{2 \mathrm{i}}$ (that may be determined by fitting Equation (1) to experimental relaxation data). By assuming that $\mathcal{M}$ is independent of $\xi_{\mathrm{i}}$ [5], for each mesh/pore class, an equation similar to Equations (13)/(14) may be written:

$$
\frac{1}{T_{2 i}}=\frac{1}{T_{2 \mathrm{H} 2 \mathrm{O}}}+2 \frac{\mathcal{M}}{\xi_{i} \sqrt{\frac{C_{0} 1-0.58 \varphi}{C_{1} \varphi}}} \quad \text { mesh, } \quad \frac{1}{T_{2 i}}=\frac{1}{T_{2 \mathrm{H} 2 \mathrm{O}}}+6 \frac{\mathcal{M}}{\xi_{i}} \quad \text { pore. }
$$

As $\xi_{\mathrm{i}}$ is the only unknown, Equation (17) allows determining $\xi_{\mathrm{i}}$ for each mesh/pore class, thus providing a deep characterization of system topology. Indeed, Equation (1) and Equation (17) allow the determination of the discrete mesh/pores size distribution $\left(A_{\mathrm{i}}, \xi_{\mathrm{i}}\right)$.

Finally, it is important to remind that the theoretical procedure presented above for the determination of mesh/pores size distribution strictly holds only in fast diffusion (or exchange) conditions occurring when water molecules mobility (represented by their self-diffusion coefficient, $D_{\mathrm{w}}$ ) is considerably higher than the relaxation rate $\left(R_{\mathrm{R}}=1 / T_{2 \mathrm{~s}}\right)$ of their protons, i.e. when:

$$
a^{2} \frac{R_{R}}{D_{w}}=\frac{a^{2}}{T_{2 S} D_{w}}=\frac{a \mathcal{M}}{D_{w}} \ll 1,
$$

where $a$ is the thickness of the bound water layer. For fibrous systems like gels, it is easy to verify that fast diffusion conditions are usually accomplished. Indeed, Chui [5] provided the following evaluation for $a$ :

$$
a \approx \frac{R_{f}}{\sqrt{\varphi}} .
$$

By remembering that, typically, $R_{\mathrm{f}} \leq 1 \mathrm{~nm}$ and $\phi \geq 0.01$, $a$ results to be lower than $10 \mathrm{~nm}\left(10^{-8} \mathrm{~m}\right)$. In addition, as, in the temperature range $20-40{ }^{\circ} \mathrm{C}, D_{\text {wf }} \sim 2.5^{*} 10^{-9} \mathrm{~m}^{2} / \mathrm{s}$ [36] and, in the same temperature range, $T_{2 \mathrm{~s}}(20 \mathrm{MHz}) \geq 0.01 \mathrm{~ms}\left(10^{-5} \mathrm{~s}\right)$, it is possible to conclude that $\mathcal{M}$ is approximately $10^{-6} \mathrm{~m} / \mathrm{s}$ and $a \mathcal{M} / D_{\mathrm{w}}$ is always lower than $4^{*} 10^{-3}$, i.e. $\ll 1$. Unfortunately, for porous systems such as some hydrogels and scaffolds, where pores size may range from 1 up to $1000 \mu \mathrm{m}$, fast diffusion conditions may not be always met. This implies that the process of protons relaxation of each class of pores may no longer be described by only one relaxation time, but more (theoretically infinite) relaxation times are required. Thus, Equation (1) should be replaced by [1]:

$$
I(t)=\sum_{i=1}^{m} A_{i} \sum_{j=1}^{\infty} I_{i j} \exp \left(-t / T_{2 i j}\right), \quad\left(\frac{1}{T_{2}}\right)_{m}=\sum_{i=1}^{m} A_{i} \sum_{j=1}^{\infty} I_{i j} / T_{2 i j},
$$

where:

$$
T_{2 i j}=\frac{\left(\xi_{i} / 2\right)^{2}}{D_{w} X_{i j}} \quad \text { and } \quad I_{i j}=\frac{12\left(\sin \left(X_{i j}\right)-X_{i j} \cos \left(X_{i j}\right)\right)^{2}}{X_{i j}^{3}\left(2 s X_{i j}-\sin \left(2 X_{i j}\right)\right)}
$$


being $X_{\mathrm{ij}}$ the positive roots of the following equation:

$$
1-X_{i j} \cot \left(X_{i j}\right)=\frac{\mathcal{M} \xi_{i}}{2 D_{w}} \text {. }
$$

Consequently, Equations (14) and (17) become, respectively:

$$
\begin{aligned}
& \left(\frac{1}{T_{2}}\right)_{m}=\sum_{i=1}^{m} A_{i} \sum_{j=1}^{\infty} \frac{I_{i j}}{T_{2 i j}}=\frac{1}{T_{2 \mathrm{H} 2 \mathrm{O}}}+6 \frac{\mathcal{M}}{\xi_{a}}, \\
& \left(\frac{1}{T_{2 i}}\right)_{m}=\sum_{j=1}^{\infty} \frac{I_{i j}}{T_{2 i j}}=\frac{1}{T_{2 \mathrm{H} 2 \mathrm{O}}}+6 \frac{\mathcal{M}}{\xi_{i}} .
\end{aligned}
$$

As $T_{2 \mathrm{ij}}$ are not independent of each other (on the basis of Equation (21), it follows $T_{2 \mathrm{ij}}=T_{2 \mathrm{i} 1}\left(X_{\mathrm{i} 1} / X_{\mathrm{ij}}\right)^{2}$ ), Equation (20) fitting parameters are $T_{2 i 1}, A_{i}$, and $M R D_{\mathrm{i}}\left(=\mathcal{M} \xi_{\mathrm{i}} / 2 D_{w}\right)$ for a total of $3 m$ parameters. Once these parameters are known by fitting Equation (20) to experimental data, Equation (23) may be employed to evaluate $\mathcal{M}$. In turn, Equation (24) may evaluate $\xi_{\mathrm{l}}$, so that the discrete pores size distribution $\left(A_{\mathrm{i}}, \xi_{\mathrm{i}}\right)$ may be obtained.

\section{Results and Discussion}

Halib et al. [31] applied the LF-NMR characterization to a porous hydrogel composed of acrylic acid and bacterial cellulose extracted from nata de coco. The gel was realized by dispersing cellulose powder in water $(1 \% \mathrm{w} / \mathrm{v})$ and, then, by adding acrylic acid in a ratio $1: 4(\mathrm{w} / \mathrm{w})$ compared to cellulose. The mixture was poured into a plastic container (size $12 \times 12 \times 1 \mathrm{~cm}$ ) and, then, irradiated in the air with an electron beam of $35 \mathrm{kGy}$ intensity ( $5 \mathrm{kGy}$ per pass, EPS 3000, Japan). The freshly prepared gel was dried and then left to swell at $37{ }^{\circ} \mathrm{C}$ in distilled water for $48 \mathrm{~h}$ to reach the equilibrium swelling. LF-NMR characterization (Bruker Minispec mq20 $(0.47 \mathrm{~T}, 20 \mathrm{MHz})$ ) was performed by measuring the water protons transverse relaxation time inside the hydrogel $\left(T_{2 \mathrm{~m}}\right)$ and the dependence of the water self-diffusion coefficient inside the hydrogel $\left(D_{\mathrm{w}}\right)$ on the diffusion time $\left(t_{\mathrm{d}}\right)$. In the case of relaxation experiments, CPMG sequence $\left\{90^{\circ}[-\tau\right.$ $180^{\circ}-\tau$ (echo) $\left.]_{\mathrm{n}}-T_{\mathrm{R}}\right\}$ with an 8.36- $\mu$ s wide $90^{\circ}$ pulse, $\tau=250 \mu \mathrm{s}, T_{\mathrm{R}}$ (sequences repetition rate) equal to $5 \mathrm{~s}$ and 36 repetitions was adopted. $D_{\mathrm{w}}$ determination was achieved by the execution of PGSE sequence (performed in triplicate at $37^{\circ} \mathrm{C}$ ) where the two gradients (duration $\delta=500 \mu \mathrm{s}$ ) occurred, respectively, $100 \mu \mathrm{s}$ after the application of the first and the second pulse.

Figure 3 displays Equations (16) and (20) best fit to experimental diffusion $\left(D_{\mathrm{w}}\right)$ and relaxation data (FID $=I(t))$. While Equation (16) best fit provides $\xi_{\mathrm{a}}=14.6 \mu \mathrm{m}$, Equation (20) best fit allows concluding that four classes $\left(A_{1}=38 \%, A_{2}=47 \%, A_{3}=9 \%, A_{4}=6 \%\right.$ ) are required to describe FID decay. In addition, it is noticeable that, in the second summation of Equation (20), for each class, the first relaxation time $\left(T_{2 i 1}\right)$ always played a predominant role $\left(l_{\mathrm{i} 1} \sim 1\right)$. Finally, the use of Equations (23) and (24) allowed concluding that $\mathcal{M}=1.1 * 10^{-5} \mathrm{~m} / \mathrm{s}$ and that pores dimension may be subdivided into the following four classes: $\xi_{1}=91$ $\mu \mathrm{m}, A_{1}=38 \% ; \xi_{2}=33 \mu \mathrm{m}, A_{2}=47 \% ; \xi_{3}=10 \mu \mathrm{m}, A_{3}=9 \% ; \xi_{4}=1.3 \mu \mathrm{m}, A_{4}=6 \%$. Interestingly, these findings are close to those obtained by image analysis performed on environmental SEM pictures. Indeed, this analysis revealed that pores characterized by a smaller diameter than $30 \mu \mathrm{m}$ represent $4 \%$ of the whole pores volume, those with a diameter comprised between 30 and $80 \mu \mathrm{m}$ are $29 \%$, while the remaining $67 \%$ are pores characterized by a diameter ranging between 80 and $120 \mu \mathrm{m}$. Furthermore, as SEM pictures did not reveal the presence of pores close to $1 \mu \mathrm{m}$, we assumed that the fourth class identified by LF-NMR analysis simply represents the water fraction outside pores swelling the hydrogel continuous structure (meshes). By relying on this hypothesis, it could be possible to conclude that the majority of hydrogel volume ( $81 \%$ ) was constituted by micrometric pores filled by water, while only $19 \%$ of 
the whole hydrogel volume was represented by polymeric meshes swollen by water.

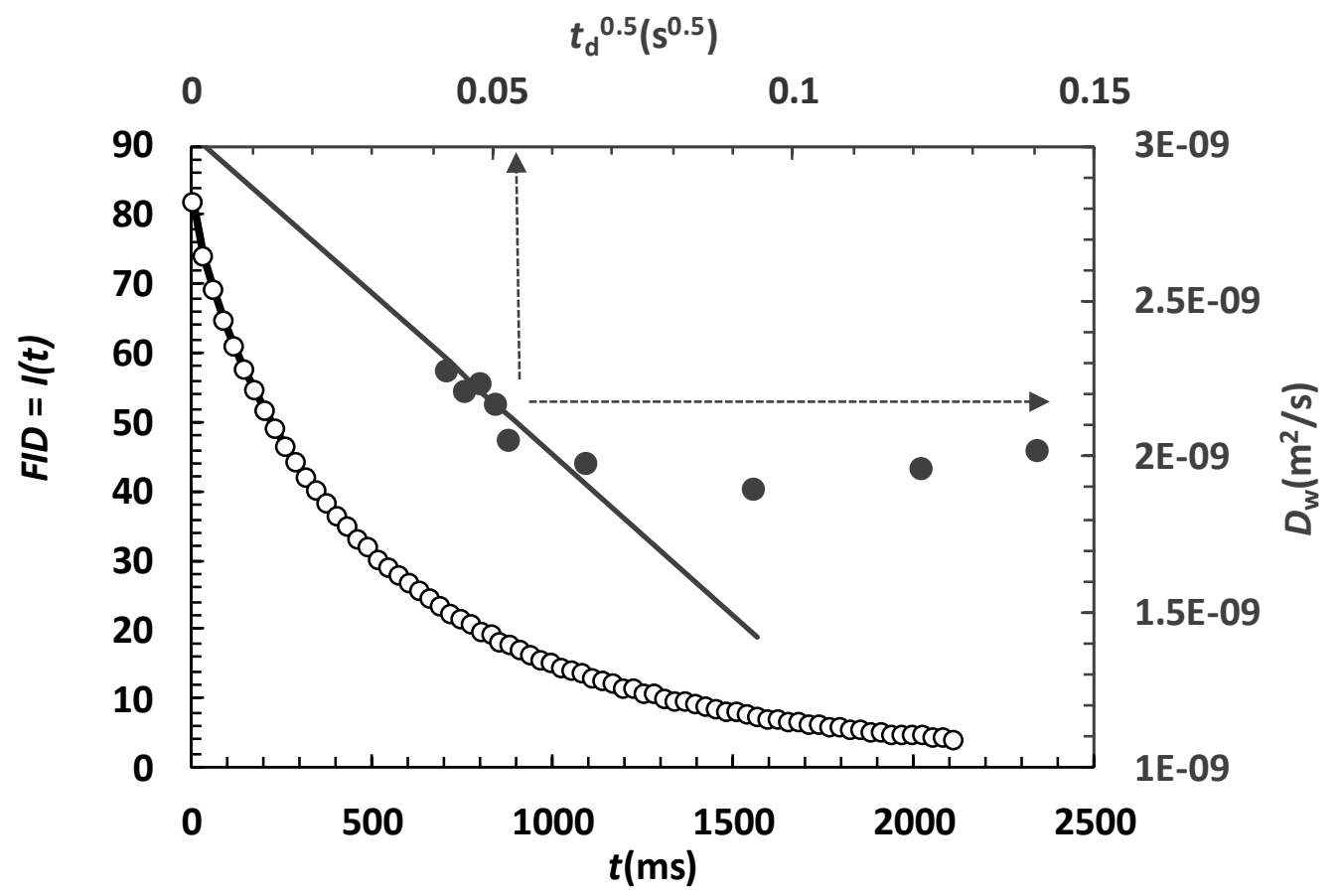

Figure 3. Porous hydrogel composed of acrylic acid and bacterial cellulose $\left(37^{\circ} \mathrm{C}\right)$. a) Left vertical axis (FID) and bottom horizontal axis (time $t$ ): Equation (20) (solid black line) best fit to relaxation data (open circles). b) Right vertical axis (water self-diffusion coefficient $D_{\mathrm{w}}$ ) and top horizontal axis (square root of the diffusion time $t_{\mathrm{d}}$ ): Equation (16) (solid gray line) best fit to experimental data (gray circles). Dashed arrows help to catch the correct vertical and horizontal axis. Adapted from [37].

Fiorentino et al. [20] focussed the attention on poly(L-lactic acid) (PLLA) scaffolds for tissue engineering applications prepared through a thermally induced phase separation (TIPS) protocol. LF-NMR characterization, performed by means of the CPMG and PGSE (the two gradients of duration $\delta=1000 \mu \mathrm{s}$ were applied, respectively, $1000 \mu \mathrm{s}$ after the first and second pulses) sequences previously illustrated, revealed that $\mathcal{M}=2.4 * 10^{-5} \mathrm{~m} / \mathrm{s}, \xi_{\mathrm{a}}=92 \mu \mathrm{m}$, and that $47 \%$ of pores showed a diameter $\xi=367 \mu \mathrm{m}, 44 \%$ $\xi=101 \mu \mathrm{m}$, and $9 \% \xi=17 \mu \mathrm{m}$. This evaluation is based, respectively, on Equations (16) and (20) best fit to experimental diffusion $\left(D_{\mathrm{w}}\right)$ and relaxation data $(\mathrm{FID}=I(t))$ displayed in Figure 4.

Interestingly, LF-NMR evaluation of pores size distribution was not so far from that descending from SEM picture evidencing pores ranging from $400 \mu \mathrm{m}$ to $100 \mu \mathrm{m}$. A further qualitative support for the LFNMR findings was provided by $\mu \mathrm{CT}$ (X-ray microcomputed tomography characterization) that revealed the presence of pores ranging from $400 \mu \mathrm{m}$ to $100 \mu \mathrm{m}$ also in the scaffold bulk. The comparison between Figure 3 and Figure 4 demonstrates that larger pores are associated with a longer relaxation process ( $2000 \mathrm{~ms}$ in Figure 3 and $~ 4500 \mathrm{~ms}$ in Figure 4) and a higher water self-diffusion coefficient. Moreover, in the case of larger pores (Figure 4), it was observed that approximately three relaxation times $\left(T_{2 i 1}, T_{2 i 2}, T_{2 i 3}\right)$ are required in the second summation of Equation (20).

Fiorentino [37], in her Ph.D. thesis, applied LF-NMR characterization (CPMG and PGSE sequences set up equal to that used for Figure 4 data) to study the time evolution of the topology of alginate/hydroxyapatite scaffolds for bone regeneration. In particular, she compared the time evolution (21 days) of the pores size distribution of empty scaffolds and of cells seeded scaffold (Osteosarcoma MG-63 human cells) filled with Dulbecco's modified Eagle's medium (DMEM). Interestingly, she noticed that the fate of smaller pores (diameter $<110 \mu \mathrm{m}$ ) was the same in empty and seeded scaffolds. This sounds reasonable as MG-63 cells 
are unable to grow in smaller pores than $100 \mu \mathrm{m}$. On the contrary, she could verify that the diameter of larger pores $(200-600 \mu \mathrm{m})$ decreased more rapidly in seeded scaffold with respect to empty scaffolds and this seemed correct due to cells growth and/or to the production of extracellular matrix. Interestingly, this characterization proved to be safe for what concerns cell survival.

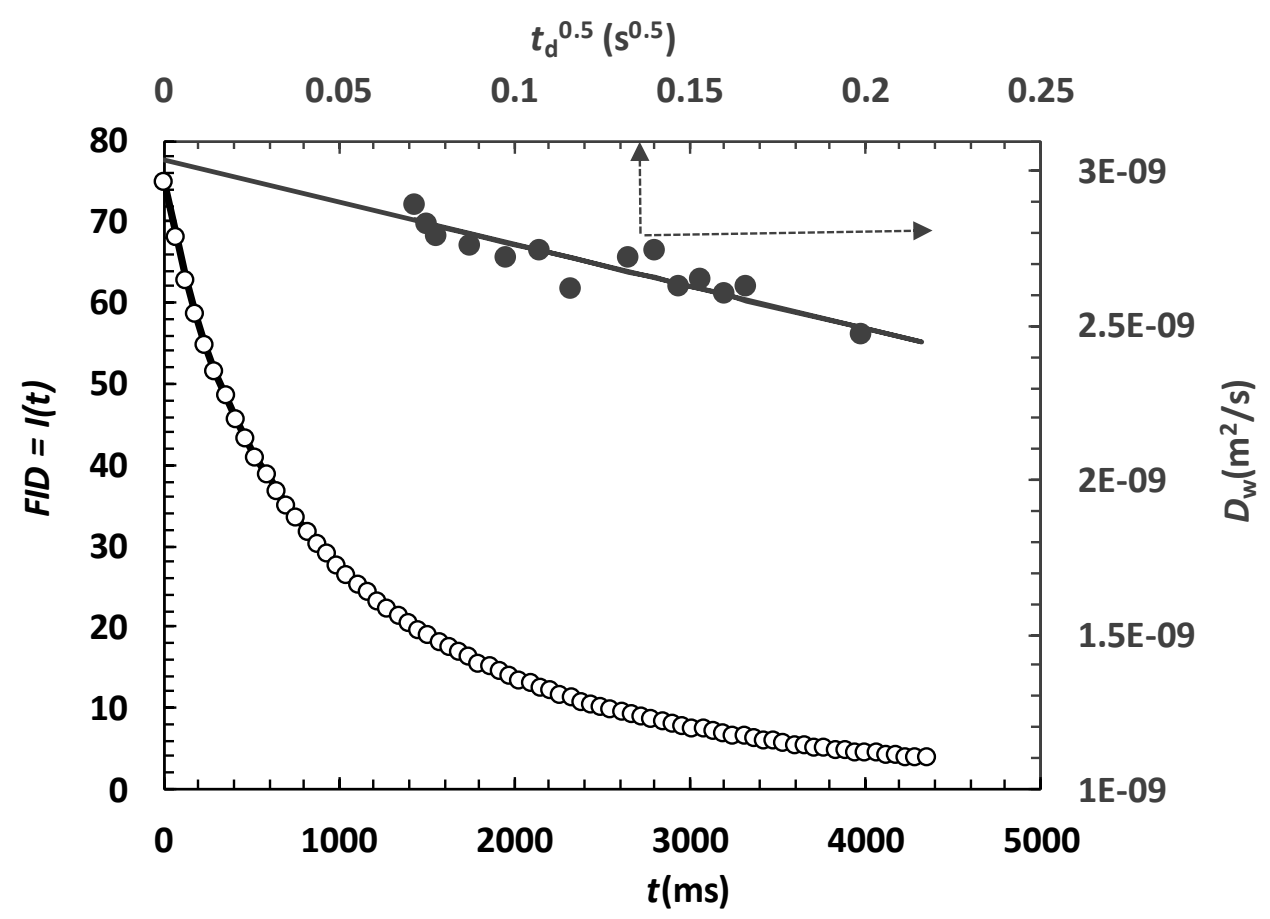

Figure 4. PLLA scaffold $\left(37^{\circ} \mathrm{C}\right)$. a) Left vertical axis (FID) and bottom horizontal axis (time $t$ ): Equation (20) (solid black line) best fit to relaxation data (open circles). b) Right vertical axis (water self-diffusion coefficient

$D_{\mathrm{w}}$ ) and top horizontal axis (square root of the diffusion time $t_{\mathrm{d}}$ ): Equation (16) (solid gray line) best fit to experimental data (gray circles). Dashed arrows help to catch the correct vertical and horizontal axis. Adapted from [20].

Abrami [38], in her Ph.D. thesis, explored the possibility of finding structural differences among samples of the femoral head withdrawn from osteoarthritis and osteoporosis patients (CPMG and PGSE sequences set up was equal to that used for Figure 3 data). Interestingly, she found that not only the average diameter of osteoarthritic samples is smaller than that of osteoporotic samples but also pores size distributions were different. Osteoporotic samples were characterized by a pores size distribution $\left(A_{\mathrm{i}}, \xi_{\mathrm{i}}\right)$ shifted towards larger pores with respect to the osteoarthritic samples one. In addition, she found that the porosity of osteoporotic samples was smaller than that of osteoarthritic samples.

Abrami et al. [27] employed LF-NMR to gain an insight into the nanostructure of a highly biocompatible hydrogel constituted by crosslinked alginate and Pluronic F127 (PF127), a synthetic poly(oxyethyleneoxypropyleneoxyethylene) tri-block copolymer able to generate micelles that organize themselves to yield a soft gel when added to water. By means of relaxation and diffusion tests, she discovered that PF127 Pluronic micelles, organizing themselves in cubic domains, induce the distortion of the alginate network so that larger meshes, filled with ordered micelles domains, coexist with smaller meshes which may host only single PF127 micelles. This result was compatible with the evidence provided by TEM pictures.

Abrami et al. [21] applied LF-NMR to establish a relation between the average relaxation time $T_{2 \mathrm{~m}}$ of the sputum of patients suffering from chronic obstructive pulmonary diseases (COPD, like cystic fibrosis) in relation to the patient's clinical conditions. This study was conducted by adopting the CPMG sequence set up described for the Figure 3 data. 


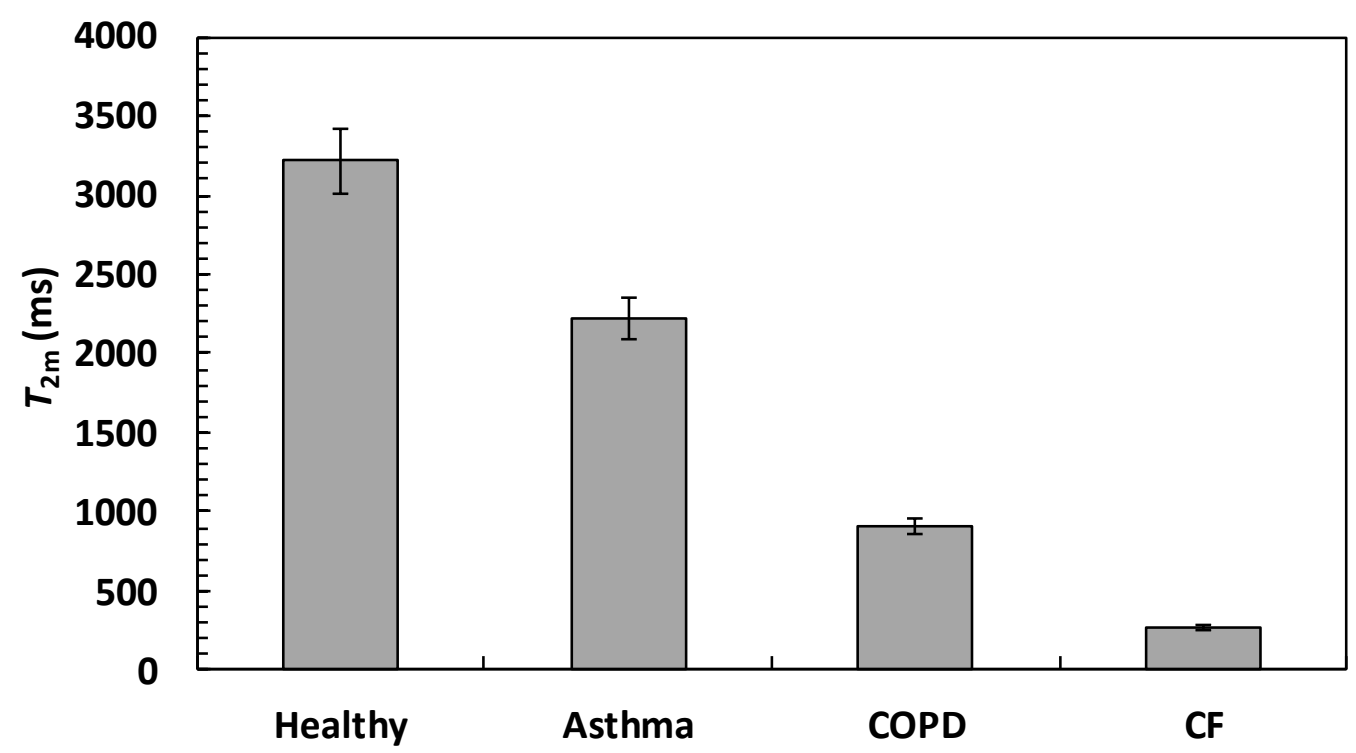

Figure 5. Sputum mean relaxation time $\left(T_{2 \mathrm{~m}}\right)$ referring to healthy subjects and three patients suffering, respectively, from asthma, COPD (lung bacterial infection), and cystic fibrosis $\left(37^{\circ} \mathrm{C}\right)$. Vertical bars indicate data standard error.

Figure 5 clearly demonstrates that, while healthy sputum is characterized by the highest $T_{2 \mathrm{~m}}, T_{2 \mathrm{~m}}$ decreases with increasing disease severity (asthma, COPD, CF). This is essentially due to the increasing presence of substances such as DNA, polymers (usually bacterial alginates), mucin, and albumin, which are typical of the considered pathologies. Indeed, all these substances act as a solid substrate that, as discussed in the "Surface effect on $T_{2 m}$ " section, induce the reduction of the water hydrogens relaxation time in a concentration-dependent fashion. It is also worth mentioning that the differences among healthy volunteers and patients are unconfined to the decreasing $T_{2 m}$ values. Indeed, Tab. 2 clarifies that, while only one relaxation time is sufficient to describe water hydrogens relaxation in healthy volunteers, the required number of relaxation times increases when considering asthma, COPD, and CF patients. This evidence is connected to the increasing heterogeneity of the solid component spatial organization inside the liquid sputum part. Thus, we may conclude that not only $T_{2 m}$ value matters for the evaluation of clinical conditions but also an equally important role is played by the relaxation spectrum characteristics $\left(A_{\mathrm{i}}, T_{2 \mathrm{i}}\right)$.

Table 2. Components of the mean sputum relaxation time $T_{2 \mathrm{~m}}$.

\begin{tabular}{|c|c|c|c|c|}
\hline & Healthy & Asthma & COPD & CF \\
\hline $\boldsymbol{T}_{\mathbf{2 m}}$ (ms) & $3223 \pm 200$ & $2225 \pm 5$ & $908 \pm 25$ & $268 \pm 2$ \\
\hline $\boldsymbol{A}_{\mathbf{1}} \%(-)$ & 100 & $84 \pm 5$ & $32 \pm 2$ & $17 \pm 1$ \\
\hline $\boldsymbol{T}_{\mathbf{2 1}}$ (ms) & $3213 \pm 200$ & $2405 \pm 144$ & $1633 \pm 98$ & $658 \pm 39$ \\
\hline $\boldsymbol{A}_{\mathbf{2}} \%(-)$ & - & $16 \pm 1$ & $32 \pm 2$ & $57 \pm 3$ \\
\hline $\boldsymbol{T}_{\mathbf{2 2}}$ (ms) & - & $1257 \pm 75$ & $794 \pm 48$ & $230 \pm 14$ \\
\hline $\boldsymbol{A}_{\mathbf{3}} \%(-)$ & - & - & $36 \pm 2$ & $26 \pm 2$ \\
\hline $\boldsymbol{T}_{\mathbf{2 3}}(\mathrm{ms})$ & - & - & $370 \pm 22$ & $103 \pm 6$ \\
\hline
\end{tabular}

A further support for the relation between LF-NMR characterization and patients clinical conditions is provided by Figure 6 showing a linear correlation between $T_{2 m}$ and FEV1 (Forced Expiratory Volume in the first second), a typical test used to evaluate patients lung clinical conditions [21].

Indeed, the correlation coefficient $r=0.836\left(t_{r}(16,0.95)<6.10\right)$ and the correlation ratio $\rho=0.901\left(t_{\rho}(16\right.$, $0.95)<8.34)$ are both statistically higher than zero and statistically equal each other $\left(F_{\text {test }}: F(7,9,0.95)>\right.$ $0.77)$. 


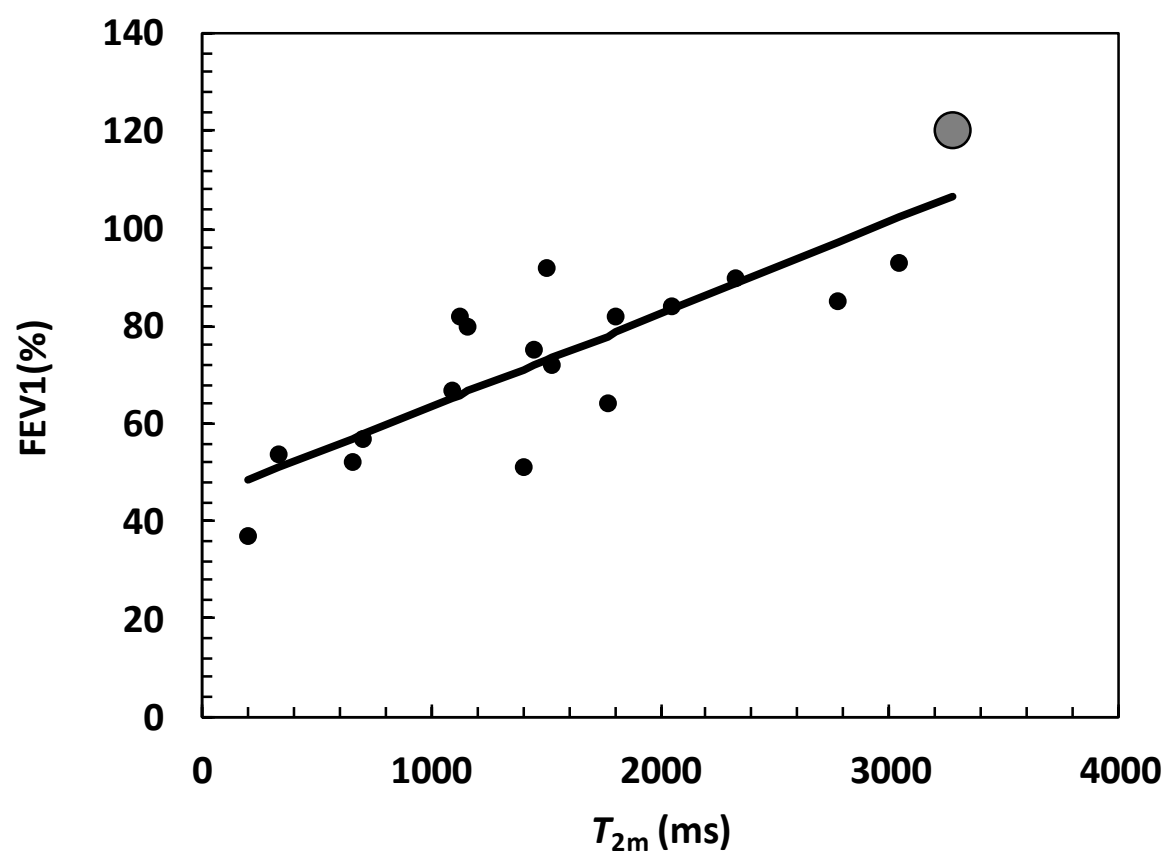

Figure 6. Relation between FEV1 (Forced Expiratory Volume in the first second) and sputum mean relaxation time $\left(T_{2 \mathrm{~m}}\right)$ referring to CF patients. Black circles represent experimental data, while the solid line is the linear interpolant. Gray large dot indicates healthy subjects.

\section{Conclusions}

We believe that we have demonstrated the potentiality of LF-NMR for the characterization of different systems such as polymeric gels, scaffolds, and biological tissues (bones and sputum of CF patients, for instance). Clearly, LF-NMR potentiality may be significantly improved by using this technique in conjunction with others such as rheology, SEM, and TEM.

Acknowledgments: This work has been supported by the "Fondazione Beneficentia Stiftung" Vaduz, by Fondo di Ateneo FRA 2016 - Trieste University, BIOFLUO project, POR FESR 2014-2020, FVG, Italy.

\section{References}

[1] K.R. Brownstein, C.E. Tarr, Physical Review A 19 (1979) 2446-2453.

[2] K.R. Brownstein, C.E. Tarr, Journal of Magnetic Resonance 26 (1977) 17-24

[3] P. Mitra, P. N. Sen, L.M. Schwartz, Physical Review B 47 (1993) 8565-8574

[4] H. C. Torrey, J. Corringa, D.O. Seevers, J. Uebersfeld, Phys. Rev. Lett. 3 (1959) 418-419.

[5] M.M. Chui, R. J. Phillips, M.J., McCarthy, Journal of Colloid and Interface Science 174 (1995) 336-344.

[6] B.P. Hills, S.F. Takacs, P.S. Belton, Food Chemistry, 37 (1990) 95-111.

[7] V. Aroulmoji, M. Mathlouthi, L. Feruglio, E. Murano, M. Grassi, Food Chemistry 132 (2012) 16441650.

[8] M.D. De'Nobili, A.M. Rojas, M. Abrami, R. Lapasin, M. Grassi, Journal of Food Engineering 165 (2015) 82-92.

[9] G.J.W. Goudappel, J.P.M. van Duynhoven, M.M.W. Mooren, Journal of Colloid and Interface Science 239 (2001) 535-542.

[10] J.P.M. van Duynhoven, G.J.W. Goudappel, G. Van Dalen, P.C. van Bruggen, J.C.G. Blonk, A.P.A.M. Eijkelenboom, Magnetic Resonance in Chemistry 40 (2002) 551-559.

[11] J. Bryan, A. Kantzas, C. Bellehumeur, SCA 39 (2002) 1-12.

[12] G. H. Sorland, K. Djurhuus, H. C. Wideroe, J.R. Lien, A. Skauge, Diffusion Fundamentals 5 (2007) 4.14.15 . 
[13] T. Stait-Gardner, S.A. Willis, N.N. Yadav, G. Zheng, W.S. Price, Diffusion Fundamentals 11 (2009) 15.115.22.

[14] M.D. Hurlimann, K.G. Helmer, L.L. Latour, C.H. Sotak, Journal of Magnetic Resonance Series A 111 (1994) 169-178.

[15] P. Fantazzini, R.J.S. Brown, G.C. Borgia, Magnetic Resonance Imaging 21 (2003) 227-234.

[16] X. Wang, Q. Ni, Journal of Orthopaedic Research 21 (2003) 312-319.

[17] C.S. Rajapakse, M.B. Zadeh, C. Li, W. Sun, A.C. Wright, F.W. Wehrli, Radiology 276 (2015) 526-534.

[18] Q. Ni, J. D. King, X. Wang, Measurement Science and Technology 15 (2004) 58-66.

[19] M. Grassi, S. Fiorentino, R. Farra, B. Dapas, G. Grassi in Polysaccharide hydrogels: characterization and biomedical applications. Pan Stanford, Singapore, chapter 4, 2015, pp. 139-166.

[20] S.M. Fiorentino, F. Carfi Pavia, V. La Carrubba, V. Brucato, M. Abrami, R. Farra, G. Turco, G. Grassi, M. Grassi, International Journal of Polymeric Materials and Polymeric Biomaterials 66 (2017) 469-477.

[21] M. Abrami, F. Ascenzioni, E.G. Di Domenico, M. Maschio, A. Ventura, M. Confalonieri, S. Di Gioia, M. Conese, B. Dapas, G. Grassi, M. Grassi, Magnetic Resonance in Medicine 79 (2018) 2323-2331.

[22] P.Y. Ghi, D.J.T. Hill, A.K. Whitaker, Biomacromolecules 3 (2002) 991-997.

[23] R. Barbucci, G. Leone, A. Chiumiento, M. E. Di Cocco, G. D’Orazio, R. Ginaferri, M. Delfini, Carbohydrate Research 341 (2006) 1848-1858.

[24] K. Hietalahti, M. Skrifvars, A. Root, F. Sundhoolmp, Journal of Applied Polymer Science 68 (1999) 671680.

[25] T. Brand, S. Richter, S. Berger, Journal of Physical Chemistry B 110 (2006) 15853-15857.

[26] M. Dobies, S. Kusmia, S. Jurga, Acta Physica Polinica A 108 (2005) 33-36.

[27] M. Abrami, I. Dagostino, G. Milcovich, S. Fiorentino, R. Farra, F. Asaro, R. Lapasin, G. Grassi, M. Grassi, Soft Matter 10 (2014) 729-737.

[28] S. Meiboom, D. Gill, The Review of Scientific Instruments 29 (1958) 688-691.

[29] D.P. Gallegos, K. Munn, D.M. Smith, D.L. Stermer, Journal of Colloid and Interface Science 119 (1987) $127-140$

[30] T. Coviello, P. Matricardi, F. Alhaique, R. Farra, G. Tesei, S. Fiorentino, F. Asaro, G. Milcovich, M. Grassi, Express Polymer Letters 7 (2013) 733-746.

[31] N. Halib, M. Amin, I. Ahmad, M. Abrami, S. Fiorentino, R. Farra, G. Grassi G, F. Musiani, R. Lapasin, M. Grassi. European Journal of Pharmaceutical Science 63 (2014) 326-333.

[32] G.W. Scherer, Journal of Sol-Gel Science and Technology 1 (1994) 285-291

[33] E.O. Stejskal, J.E. Tanner, Journal of Chemistry Physics 42 (1965) 288-292.

[34] K.R. Brownstein, C.E. Tarr, Journal of Magnetic Resonance 26 (1977) 17-24.

[35] L.L. Latour, P.P. Mitra, R.L. Kleinberg, C.H. Sotak, Journal of Magnetic Resonance A 101 (1993) 342346.

[36] M. Holz, S.R. Heil, A. Sacco, Physical Chemistry Chemical Physics 2 (2000) 4740-4742.

[37] S.M. Fiorentino, Ph.D. thesis, XXVII cycle, University of Trieste, Dept of Engineering and Architecture, 2015.

[38] M. Abrami, M. Ph.D. thesis, XXIX cycle, University of Trieste, Dept of Life Sciences, 2017.

(C2018 by the authors; licensee IAPC, Zagreb, Croatia. This article is an open-access article distributed under the terms and conditions of the Creative Commons Attribution license (http://creativecommons.org/licenses/by/3.0/) (cc)) EY 